\title{
Energy Management of the Multi-Mission Space Exploration Vehicle using a Goal-Oriented Control System
}

\author{
Julia M. B. Braman \\ NASA-Johnson Space Center \\ 2101 NASA Parkway \\ Houston, TX 77058 \\ 281-483-2277 \\ julia.m.braman@nasa.gov
}

\author{
David A. Wagner \\ Jet Propulsion Laboratory, California Institute of Technology \\ 4800 Oak Grove Dr. \\ Pasadena, CA 91109 \\ david.a.wagner@jpl.nasa.gov
}

\begin{abstract}
Safe human exploration in space missions requires careful management of limited resources such as breathable air and stored electrical energy. Daily activities for astronauts must be carefully planned with respect to such resources, and usage must be monitored as activities proceed to ensure that they can be completed while maintaining safe resource margins. Such planning and monitoring can be complex because they depend on models of resource usage, the activities being planned, and uncertainties. This paper describes a system and the technology behind it - for energy management of the NASA-Johnson Space Center's Multi-Mission Space Exploration Vehicles (SEV), that provides, in an onboard advisory mode, situational awareness to astronauts and real-time guidance to mission operators. This new capability was evaluated during this year's Desert RATS (Research and Technology Studies) planetary exploration analog test in Arizona. This software aided ground operators and crew members in modifying the day's activities based on the real-time execution of the plan and on energy data received from the rovers.
\end{abstract}

\section{TABLE OF Contents}

1 InTRODUCTION $\ldots \ldots \ldots \ldots \ldots \ldots \ldots \ldots \ldots \ldots \ldots \ldots$. 1

2 SEV AND DESERT RATS OVERVIEW .......... 2

3 SYSTEM DESIGN $\ldots \ldots \ldots \ldots \ldots \ldots \ldots \ldots \ldots \ldots . \ldots \ldots$

4 RESULTS FROM DESERT RATS .............. 5

5 Conclusion $\ldots \ldots \ldots \ldots \ldots \ldots \ldots \ldots \ldots \ldots \ldots \ldots \ldots, 6$

ACKNOWLEDGEMENTS $\ldots \ldots \ldots \ldots \ldots \ldots \ldots \ldots \ldots .6$

REFERENCES $\ldots \ldots \ldots \ldots \ldots \ldots \ldots \ldots \ldots \ldots \ldots \ldots, 6$

BIOGRAPHY $\ldots \ldots \ldots \ldots \ldots \ldots \ldots \ldots \ldots \ldots \ldots \ldots, 6$

\section{INTRODUCTION}

All space missions share a need to optimize the use of consumable resources such as fuel, oxygen, water, and stored energy because these all contribute significantly to the limited mass budget that can be delivered to a remote destination. However, because these resources are critical to the success and safety of the mission, they generally cannot be

\footnotetext{
${ }^{1}$ U.S. Government work not protected by U.S. copyright.

2 IEEEAC Paper \#1274, Version 1, Updated 26/10/2010.
}

completely consumed. Some safety margins have to be maintained at all times, and all activities must be carefully planned to ensure that these safety margins are ensured. This paper describes a prototype system developed for NASA's MultiMission Space Exploration Vehicle (SEV) that provides an onboard, real-time advisory capability for astronauts to help them manage energy stored in the vehicle's batteries.

The "control system" core of the advisory system was adapted from an earlier demonstration system developed using the Mission Data System (MDS) framework [1] for the ATHLETE rover at the Jet Propulsion Laboratory (JPL). This system features a real-time core and includes state estimators and state variable projection models along with the goal planner and executive. The advisory system communicates with the SEV using a common robotic API, allowing it to use the existing telemetry data delivery infrastructure. Separate user interface applications allow operators to propose activity plans to the planner and view detailed execution status, and existing driver console displays are used to present key status information to the crew.

The SEV's navigation display software uses Google Earth $^{\mathrm{TM}}$ as a presentation layer framework for displaying a geographical map view of the activity plan, which is specified in custom $\mathrm{kml}$ files. So, a translator that extracts the waypoint and timing data from the $\mathrm{kml}$ file and converts those into position goals and temporal constraints in a goal network representation of the plan was developed. Thus, when a user proposes an activity, two types of goals are created; the kml file generates activity-related goals and temporal constraints, and flight rules and safety constraints generate additional ones. The set of goals is then scheduled by the planner, which verifies that all temporal and state constraints are satisfied across the plan according to the projection models for all relevant states. When the plan "executes," the activity goals are achieved directly by the astronauts driving the rover, but the control system observes driving data and continually evaluates progress against the plan using its projection models. Because of this, the system reports both immediate constraint violations and violations that will occur in the future. Specifically, it can detect when the current energy consumption rate 


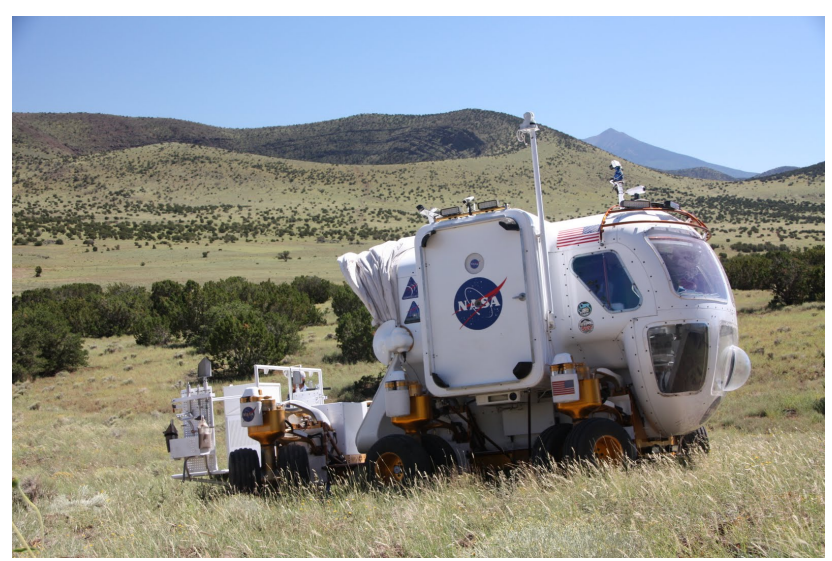

Figure 1. NASA- Johnson Space Center's Space Exploration Vehicle

is such that a margin constraint will eventually be violated.

An evaluation of this new capability was performed during this year's Desert RATS planetary exploration analog test in Arizona. Dual rover operations for a fourteen-day traverse were planned with the aid of this new tool. In the field, this software aided astronauts driving the rovers by providing energy projections, including projections of energy needed to perform contingency (rescue) procedures. It supported ground operators in modifying the day's activities based on the real-time execution of the plan and on energy data received from the rovers.

This operationally useful capability is an innovative use of a goal-oriented control framework that was originally intended for autonomous embedded robotic control systems. The Mission Data System frameworks provide easily adapted interfaces for modeling the target system, its constraints and activities, and integrated capabilities to further automate humanin-the-loop plan creation and repair.

\section{SEV AND DESERT RATS OVERVIEW}

NASA's Multi-Mission Space Exploration Vehicle, shown in Figure 1, is a next-generation modular concept vehicle intended to be used in a variety of target environments including the moon, Mars, or even an asteroid [2]. The current generation consists of an enclosed cabin mounted on a wheeled chassis. The cabin is designed to allow two crew members to live and work in shirt sleeves for up to two weeks; two spacesuit ports enable the crew to easily exit the cabin for extra-vehicular activity (EVA). The chassis features a crab design, allowing movement in any direction including 360 degree point turns. Each SEV is powered by lithium-ion batteries with a specific energy of $125 \mathrm{~W}-\mathrm{hr} / \mathrm{kg}$ [2]. The requirements for a flight version of the SEV call for batteries with a specific energy of $200 \mathrm{~W}-\mathrm{hr} / \mathrm{kg}$.

The SEV has been an integral part of the Desert RATS space surface operations analog test for the past three years. The
2010 Desert RATS test had several objectives that pertained to the operation of two SEVs, each with two crew members. The crew spent seven days and nights in the rovers and covered nearly $20 \mathrm{~km}$ per day. Several different methods of operation were compared, including continuous communication versus twice-a-day communication with the mission control center, as well as leader-follower traversing versus a divideand-conquer method [3].

One of the important operations concerns with the different traverse styles was contingency planning. It would be important on a real mission to consider rescue scenarios if one of the rovers became disabled. These rescue scenarios, or contingency plans, drive the implementation of flight rules for the analog test. These flight rules basically constrain the rovers to have enough power to rescue the other rover given two different rescue scenarios at all times. In order to implement this type of control, a consumables model would be necessary, as well as detailed plans for both rovers. Given the twice-a-day communication paradigm, this knowledge must be automatically calculated so that it is available to the crew at any given time during their traverse.

During the Desert RATS test, the system that will be described here calculated the appropriate contingency times, which were the times to complete the two contingency paths, including the necessary battery charge time and crew and supply transfer times; the drive time, which was the maximum time that the rover could drive given the most constraining consumable; and the hold-and-wait time, which was the maximum time the crew could survive in the stationary rover with only the essential systems powered, given the most constraining consumable.

\section{SYSTEM DESIGN}

\section{Requirements and Constraints}

Space operations must be designed to optimize the use of various resources including fuel, oxygen, and stored energy. The cost and risk of getting those resources into space, along with the tanks, batteries, and other storage systems needed to support them, are among the primary obstacles to human space exploration. Efficient use of resources implies a need to operate close to safety margins. Doing that safely when the resource usage is dynamic requires careful management of the resources. Operations must be planned in advance to fit within resource budgets, and then executed according to the plan.

Due to the complex and interconnected nature of the missions and the resources, mission planning is not an open-loop process. Operational flexibility is needed to accommodate exploration, uncertain environments, and the possibility of failures. Models of resource usage must be a function of the plan, and these models must be updated as the plan changes so that safety constraints can be monitored in real-time. Because the production and consumption cycles are complex, no model 
will account for everything, but it is critical that the resource is not exhausted during operations. So, constant reprojection of the models over the updated plan is imperative to ensure safety constraints will always be enforced.

When designing this system for the SEV, consideration was given to where it would be most useful. Traditionally, this sort of oversight is performed in mission control (on earth). For the system to maintain precise knowledge of resource consumption requires mostly continuous access to measurements of consumption and production rates. The anticipated lunar surface operations near the poles could make communications with the ground intermittent. In the field test environment, communications are even worse. So for the system to be able to continually monitor dynamic resource use and provide the earliest possible warning to astronauts requires that the system run onboard. In this demonstration project the system ran on a computer in the SEV's chassis.

\section{Goal-Oriented Control and the Mission Data System}

The Mission Data System (MDS) [1] was designed as a general-purpose framework for robust control systems with this type of problem in mind. MDS provides a framework for modeling activity plans as constraint networks, and has a planning and execution engine that can verify constraint satisfaction at plan time and at run time through a process of automated real time state projection.

In this system MDS was used in an advisory mode where the astronauts exercise control external to the software system. This "control system" is merely monitoring execution of the plan in order to enforce safety constraints. As described in [4], it makes sense to view this as a control system where the system models the astronauts as smart actuators. Its recommendations are interpreted by the astronauts as advice, but modeled in the system as commands [5].

\section{System Energy Model}

Figure 2 depicts a simplified model of system states relevant to the battery energy state. To simplify the analysis and implementation only a few key producers and consumers of electrical power were included individually in this model. Ovals identify the system state variables of interest, and arrows indicate state effects (e.g., power consumption affects the battery state of charge). The triangles indicate measurements the system could produce as evidence for estimating states.

Four major power consumers were considered in addition to the solar panels (simulated by outboard generators), which are the only energy producer. The largest consumer of power is the mobility system that includes the driving motors. Since these motors consume energy roughly as a function of distance driven, and a separate onboard navigation system was able to provide position and velocity, this aggregation simplified the analysis while providing reasonably good precision.

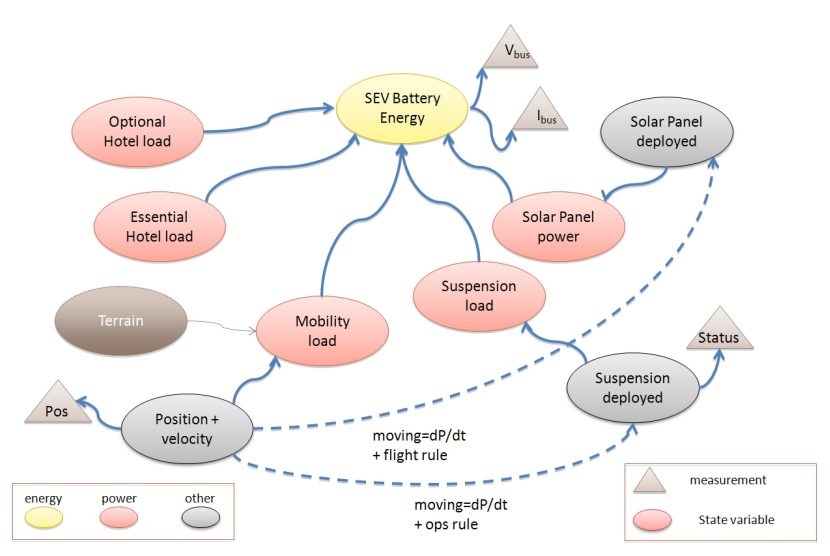

Figure 2. Energy state effects diagram

The active suspension motors were modeled as a separate group so that the model could reflect the policy to power the suspension only while driving, and turn it off when parked. All of the avionics and life support subsystems (called hotel loads) were modeled in two groups: one that is considered essential, and thus always present, and a second optional group that could be powered off to save energy in an emergency. This latter group enabled the system to consider discrete alternate optimal and sub-optimal tactics.

The dashed lines in the figure indicate policy-based state effects. The model enforces a flight rule that the simulated solar panels must be stowed while driving (and generating no energy), and an operational rule that says they will always be deployed when parked. A similar rule is enforced for the suspension. Such procedural effects create uncertainty in the projection models because the system cannot predict the precise timing of astronauts following these procedures.

Although mobility energy is roughly consumed as a function of distance driven, the condition of the surface (hard or soft, or size of rocks) will also have a significant effect. Similarly, the surface slope (change in potential energy) will have a noticeable effect. These effects were omitted from the initial model to keep the work in scope, but will be included in future updates.

\section{Activity Plan Model}

Activities are planned using a visualization system based on Google Earth (Figure 3) which allows scientists and mission planners to select observation sites on map overlays and then select approximate driving paths from one site to the next. Prototype plans can then be verified using a ground-based planning system that uses the same models as the advisory system.

The xml-based output of this process (a Google Earth KML file) is used as the operational plan description for onboard visualization and as an input to the advisory system. This format provides only the location of each waypoint and the duration allowed for making observations and other activities 


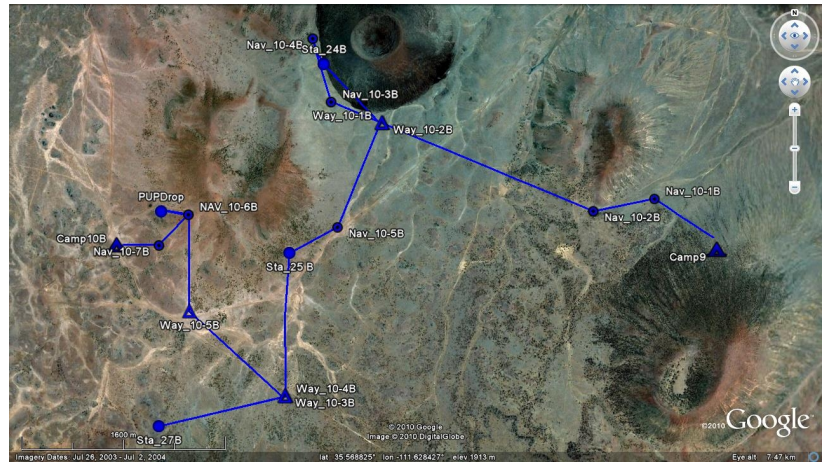

Figure 3. Example traverse map in Google Earth

at that location.

\section{Mapping Activities to Goals}

The advisory system ingests this data and elaborates each location into a position goal (be at the location for the given duration) along with a preceding driving goal to move to that location. Mobility power goals reflecting model expectations are elaborated as a function of whether the vehicle was to be parked or moving across each interval. Additional goals are elaborated for the remaining power states reflecting the policies for operating each subsystem. For example, the solar panel goals reflect the policy that they will be stowed while driving and deployed while parked.

\section{Crew and Mission Operations User Interfaces}

The outputs of the MDS advisory system are organized for consumption by the crew and mission control as a page in the SEV's drive display graphical user interface (GUI). A screenshot of this GUI page is shown in Figure 4. The GUI is comprised of four basic sections: the energy projection graph, replan options, contingency flight rule times, and the energy gauge.

The Energy Projection graph shows the maximum and minimum projected battery energy values at each future time point in the active plan. The uncertainty in the energy model causes these lines to diverge over time. Also displayed on the graph is the minimum battery energy constraint, the value of which is given to the advisory system as a maintenance safety constraint based on a flight rule. This graph is updated by the advisory system every ten seconds.

The replan options section consists of the Execution Status in the upper right corner, the Messages box directly under that, and the Replan Options box under the Energy Projection graph. As long as the actual and the minimum projected energy constraints stay above the flight rule minimum energy constraint, the execution status is nominal, there will be no messages, and the Replan Options box will not be populated, as shown in Figure 4. However, if an actual or projected failure is detected, the execution status will change to "Replan Necessary" (actual failure) or "Possible Replan Needed"

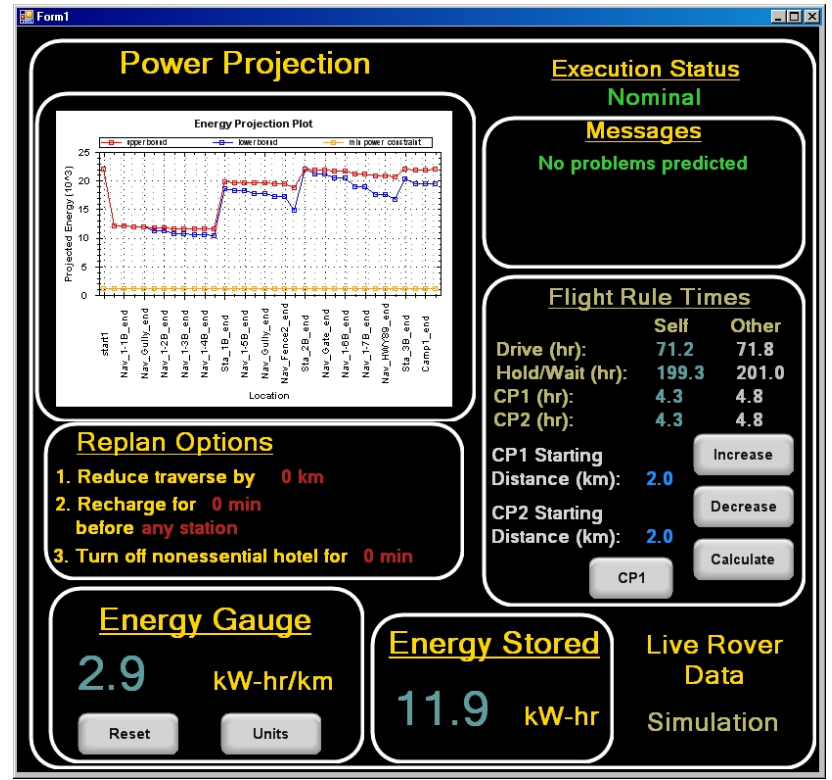

Figure 4. Screenshot of the energy management GUI

(projected failure). The Messages box then displays the point in the plan at which the failure has been detected, and the Replan Options will be populated given the energy discrepancy at the point of failure. Three different options are calculated; first, the distance by which travel should be reduced is calculated given a model that assumes the SEV travels at a nominal speed of $1 \mathrm{~km} / \mathrm{hr}$. Second, the amount of recharge time that should be added and when it should be added is calculated, and finally, the amount of time that the non-essential hotel load is removed to account for the energy discrepancy is displayed. These numbers are meant as a starting point for mission operators or crew members who will be creating a new plan to account for the energy difference. An example of a failed plan is shown in Figure 5.

The contingency flight rule times can be found on the right side of the GUI. While the rest of the GUI is listening to an energy advisory system that takes in actual data from the rover, this section is different. Because of the reduced capacity of the batteries that are currently in the SEVs compared to the requirements for the flight version, this section of the GUI listens to a simulated energy advisory system that is running models for the flight version of the SEV. This simulated system takes in the same plan as the live rover data version, but uses different energy models for projection and simulation of the vehicle's energy response.

Accurate contingency path distances are a function of both rovers' positions, paths, and the location of the battery charging apparatus, which may be separated from the SEV. Because the minimum distance path between the two SEVs may not be traversable, a safe contingency path involves backtracking via known path to an intersection point and following the planned path of the disabled SEV until it is found. Because of this, calculating contingency path distances is com- 


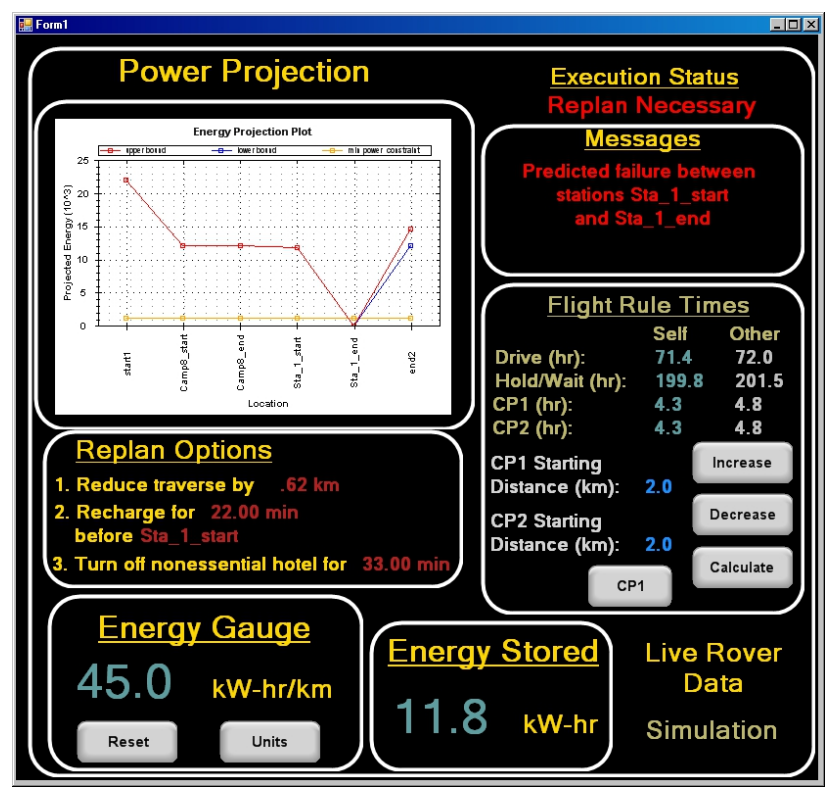

Figure 5. Screenshot of the energy management GUI when the plan has failed

plex and requires a model of the combined dual SEV system. Currently, the model handles each SEV and its path independently, so a simplified model of the contingency path lengths is used. The path lengths are estimated by adding a crewentered starting distance to the distance accumulated during the day's traverse. The buttons along the edges of the box allow the crew to easily adjust the starting distance for the day if the test plan called for it. The flight rule times are updated only when the "Calculate" button is pushed to avoid having the times change while the crew is reporting them back to mission control or to the other SEV. Two sets of flight rule times are displayed; the first are the times for the SEV that the drive display controls, and the other set are estimated times for the other SEV, given its plan for the day and a model of its power characteristics.

Finally, the energy gauge part of the GUI consists of two boxes along the bottom edge, the Energy Gauge and the Energy Stored boxes. The Energy Stored box simply displays the current estimate of the actual rover's battery capacity, updated every ten seconds from the energy advisory system. The Energy Gauge consists of a resettable infinite filter relating energy usage and distance traveled. One is able to invert the units of the Energy Gauge, based on preference. The energy and distance data used to populate the Energy Gauge box is updated every ten seconds from the energy advisory system. Altogether, the four pieces of the energy management GUI serve to inform the crew and the mission operators what the energy state of the vehicle is and how to update the plan if that energy state may in the future violate any constraint.

\section{RESUlTS FROM DESERT RATS}

The energy advisory system and accompanying GUI display influenced how the Desert RATS test was conducted, both from the crew and the operations perspective. First, the projection capability of the energy advisory system was used during traverse planning before the field test. As plans were designed, they were scheduled in the energy advisory system in a simulation mode, and the resulting projection graph was examined for potential problems due to battery energy. As a result, the traverse plans were sufficiently conservative with respect to energy.

In the field, the operations crew used the energy advisory system in several ways. First the advisory system's energy estimate was corroborated with other energy models, including the battery management system's state of charge measurement and the engineers' intuition and experience with the battery's capacity based on voltage. This system became very important when it was realized that the new power converters allowed the vehicle to charge much faster than expected. The operations crew then decided not to charge at every opportunity as dictated in the flight rule, and used the energy projection graph on the energy management GUI to choose when to charge and when not to. This approach worked very well, as there were no schedule changes due to the battery's state of charge.

The crews in the vehicles, in addition to using the energy management GUI for the contingency path times, often looked at the GUI for the energy stored and energy gauge data. Comments during the test indicated that these pieces of information, in addition to the energy projection graph, gave the crew more situational awareness; in the past, the SEV's state of charge was essentially a black box to the crew. The crew also visited this GUI page in order to check their contingency path times to fulfill a flight rule that required them to communicate these numbers to either mission control or the other vehicle's crew before each EVA or every two hours. The intent of this flight rule was not to cause the crew to perform any contingency procedures or have the day's traverse in any way affected by these numbers, but instead it was to make the crew and the mission operators aware of the need for these sorts of rules and procedures. It was for this reason that a model of the flight version of the SEV generated the contingency path numbers instead of the analog test SEV model.

The energy advisory system worked very well for the Desert RATS field test. The software was flexible enough to handle the discrepancies and delays that crept into the schedule as it continued to put out energy estimates while keeping track of where the plan should be. This was useful, as one could see how these disturbances affected the projected energy usage given the day's nominal plan. The only failure experienced with this system was due to the contingency path times' dependence on the distance actually traveled, which was pulled from a position estimate calculated by another piece of software. This could be mitigated in the future by better understanding the uncertainty in the position estimation within the advisory system. 


\section{Conclusion}

The energy advisory system described in this paper predicted expected energy usage in the system given models of the system and plans of operations. This energy advisory system was run on board the SEV and evaluated at the Desert RATS analog test. While this software was not critical to mission success, it allowed operators to relax safety margins (such as the flight rule that dictated charging at every stop) in a safe manner because of the more accurate energy models it employed. This software also allowed the crew more situational awareness and visibility into energy management, which was something only the mission operations team had in the past.

Using MDS as the underlying engine provided a clean and efficient way to model the resources and their affecting states as a function of the activity plan. Adapting the models was completed very quickly with most of the development effort going into calibrating and testing the models onboard, and tuning the user interfaces.

Future work includes many upgrades to the current model to extend its usefulness for monitoring flight rules and safety constraints in upcoming analog testing. First, the model would be upgraded to include both SEVs and their plans. Communications between the SEVs could then be tracked and the maximum time between communications could be constrained, according to an existing flight rule. Next, the removable battery charging apparatus would be included in the model, as well as a sunlight model. This and the dual SEV model would allow for more accurate calculations of the contingency path distances and times. Simple models of other consumables, such as oxygen and water, would also be included to make the drive and hold-and-wait contingency times more correct. The terrain features like roughness and height could be included in the model to improve the prediction of mobility power usage, which is the greatest cause of uncertainty in the current model. Finally, the battery energy storage model could be improved from the simple linear model currently used to give the energy projection more fidelity.

\section{ACKNOWLEDGMENTS}

Part of this research was carried out at the Jet Propulsion Laboratory, California Institute of Technology, under a contract with the National Aeronautics and Space Administration. Thanks are due to Dan Dvorak, Greg Horvath, David Mittman, and Jay Torres on the JPL side, and Stanley Lewis, Aaron Hulse, Mike Gernhardt, Andrew Abercromby, Jodi Graf, Rob Burridge, Kim Hambuchen, and Bill Bluethmann from JSC.

\section{REFERENCES}

[1] "Mission Data System.” http://mds.jpl.nasa.gov, June 2010.

[2] "NASA's Space Exploration Vehicle."
http://www.nasa.gov/exploration/home/SEV.html, August 2010.

[3] "NASA's Desert RATS." http://www.nasa.gov/exploration/analogs/desert_rats.html, September 2010.

[4] D. Wagner, D. Dvorak, L. Baroff, A. Mishkin, M. Ingham, M. Bennett, and D. Mittman, "A control architecture for safe human-robotic interactions during lunar surface operations," in AIAA Infotech@Aerospace Conference Proceedings, April 2009.

[5] D. Wagner, D. Dvorak, A. Mishkin, G. Horvath, G. Johnson, and G. Jones, "Architectural concepts for humanrated automation," in AIAA Infotech@ Aerospace Conference Proceedings, April 2010.

\section{BIOGRAPHY}



Julia Braman is a robotics system engineer in the Robotic Systems Technology Branch at NASA-Johnson Space Center in Houston, TX. She develops autonomous control systems for the various robotics projects in her group, including the SEV. Julia has a BS in Mechanical Engineering from Purdue University, a MS in Mechanical Engineering from the California Institute of Technology, and earned her $\mathrm{PhD}$ in Mechanical Engineering from Caltech in 2009.



Dave Wagner is a software system engineer and architect in the Flight Software Applications and Data Management group at JPL and was a principal developer of the Mission Data System in 2000-2006. Since then he has continued to apply MDS technology in demonstration projects such as this. He has a BS in Aerospace Engineering from the University of Cincinnati, and MS in Aerospace Engineering from the University of Southern California. 